\title{
Imaging of self-assembly and self-assembled materials
}

\author{
P. V. Braun
}

Department of Materials Science and Engineering, University of Illinois at Urbana-Champaign, Urbana, IL 61801

Self-assembled materials have recently been of great interest to a wide range of scientists for multiple, and indeed quite disparate applications. Self-assembly has been proposed as a route to photonic band gap materials, semiconductor devices, molecular sieves, biomaterials, and molecular monolayers. For many of these processes, there are few very good routes to image the self-assembly process, or even the structure of the final product. Because we are generally interested in three dimensional systems, visualization of the process becomes even more difficult. Furthermore, we often desire to obtain structural information on the nanoscale, and finally, many of our systems are solution based, and thus not amenable to conventional high vacuum imaging procedures.

We utilize a range of techniques to obtain morphological information on our systems. These include single photon confocal microscopy and multiphoton microscopy, and conventional transmission electron microscopy. Depending on the system, three dimensional structural information can be obtained from either holography or reconstruction of serial sections. We utilize confocal microscopy to investigate two different material systems, and for real time visualization of the assembly process. The first use we have for confocal microscopy is for self-assembled membrane materials, where we are determining the three dimensional pore structure of the membrane (figure. 1), and the second is for photonic band gap materials (figure 2), where the key aspects are to determine the assembly process and resulting three-dimensional structure, and to create defined defects into the structure. It is these defined defects that allows for the fabrication of waveguides and optical cavities (figure 3).

Both the membrane and photonic band gap material studies are performed on a Leica confocal microscope. The core unit of the self-assembled photonic band gap materials are monodisperse colloidal particles. Under the appropriate conditions, these particles can be crystallized into threedimensional materials with long range periodicity with a lattice constant similar to the wavelength of the light to be modulated. The assembly process takes between minutes and hours, which is much slower than the frame rate of the microscope. Thus real time imaging is possible. The multiphoton imaging is performed using a mode locked Ti-sapphire laser, this same laser is used to write defects into the self-assembled colloidal crystals (figure 3 ).

Most of the electron microscopy is performed on a Philips CM12 or CM20. In the electron microscopy experiments, we are imaging the structure of semiconductor nanostructures formed through growth in a self-assembled matrix (figure 4). Here, a soft template, specifically a non-ionic hexagonal lyotropic liquid crystal, is used to control the growth of a hard, inorganic phase, in this case CdS. The growth process in some aspects resembles the biomineralization process, as biominerals such as bone or shell are also formed in three dimensional spaces defined by selfassembled biomolecules [1].

[1] This work supported in part by the US DoE and the NSF. 


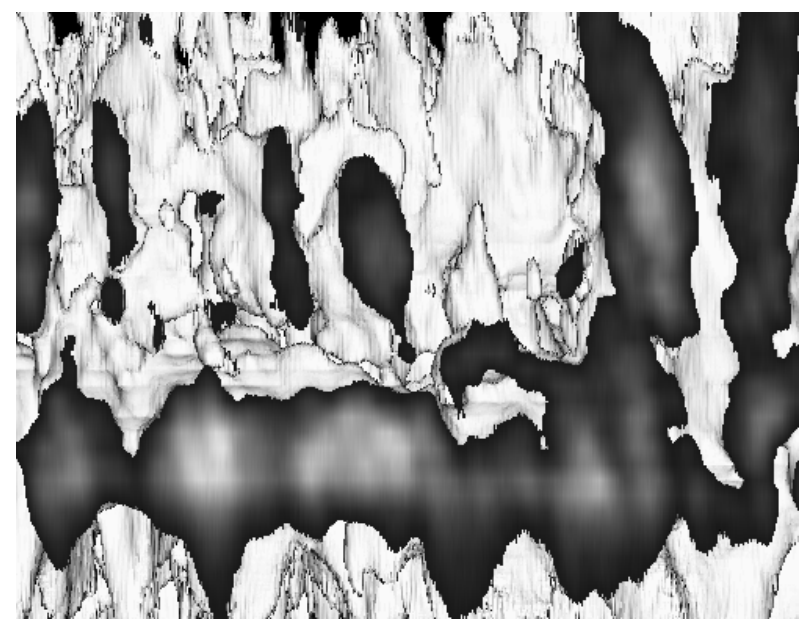

Figure 1. Three dimensional reconstruction of a membrane generated from 60 slices.

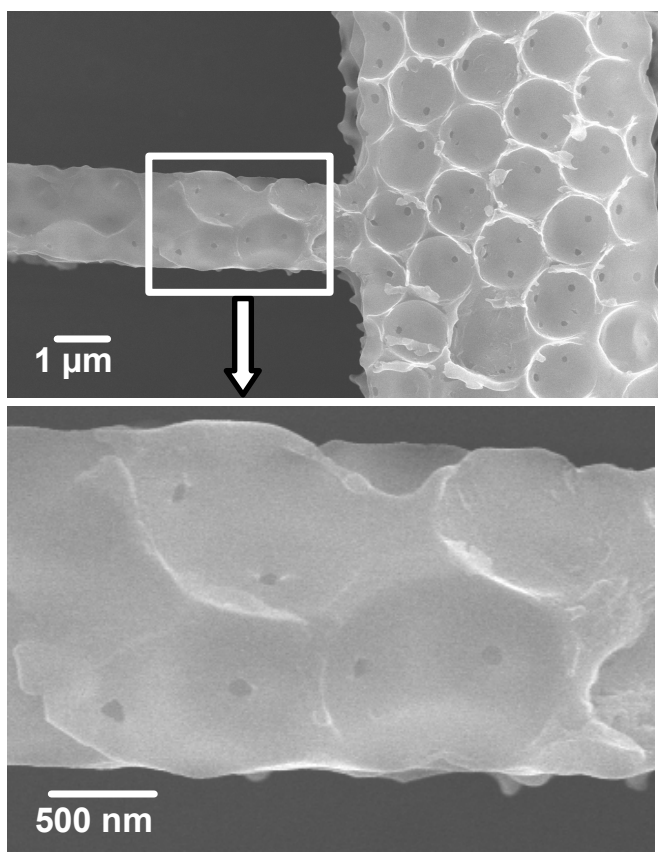

Figure 3. SEM micrograph of polymer feature formed through three-photon polymerization within a silica colloidal crystal. The silica has been removed with HF, exposing the polymer.

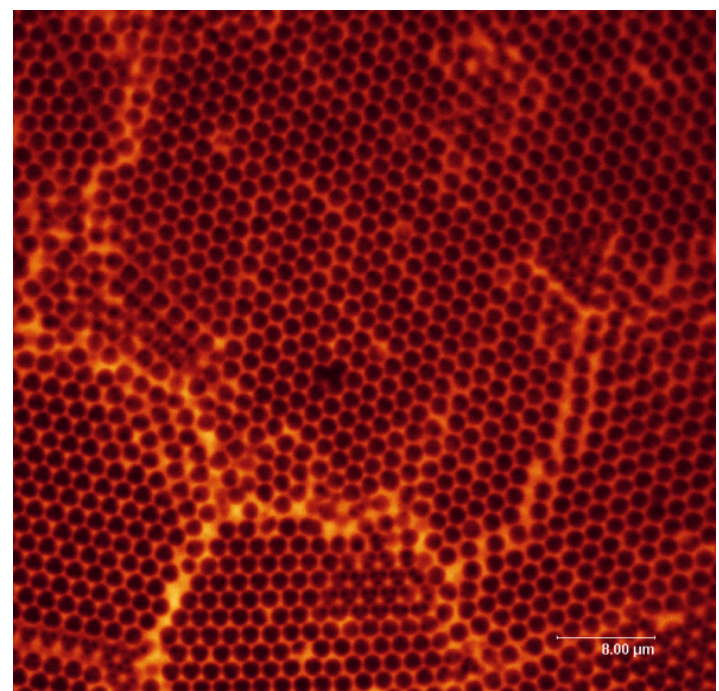

Figure 2. Silica colloidal crystal formed from $1.6 \mu \mathrm{m}$ diameter silica colloidal spheres. Space between spheres filled with $1 \times 10^{-4}$ molar Rhodamine-6G in DMF, Excitation wavelength: $488 \mathrm{~nm}$, fluorescence widow: 520 to $580 \mathrm{~nm}$.

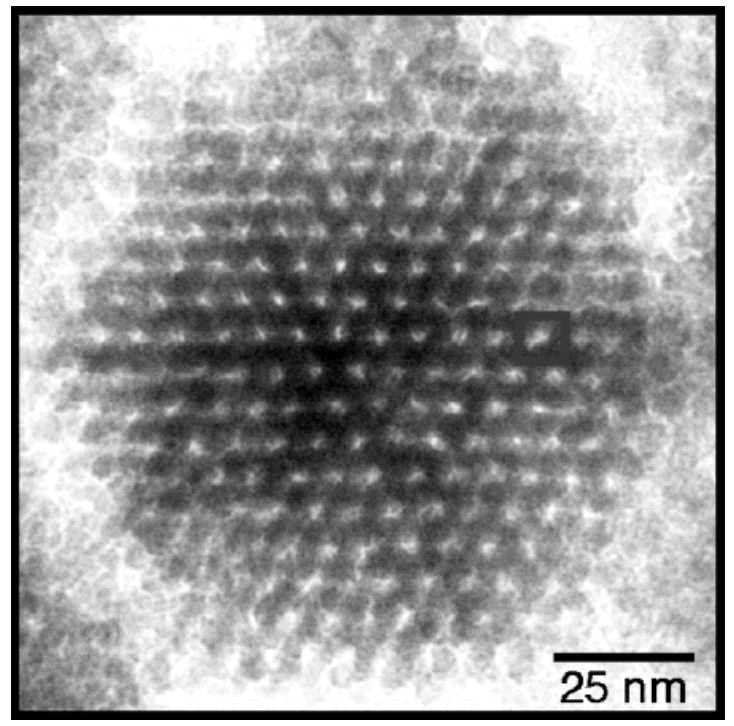

Figure 4. CdS nanostructure formed through templating with a hexagonal lyotropic liquid crystal. 